\title{
Isopistonic angle for multi-aperture interferometers from isoplanatic angle
}

\author{
T. Elhalkouj ${ }^{1}$, A. Ziad ${ }^{2}$, R. G. Petrov ${ }^{2}$, M. Lazrek ${ }^{1}$, Y. Elazhari ${ }^{3}$, and Z. Benkhaldoun ${ }^{1}$ \\ 1 Université Cady-Ayyad Faculté des sciences LPHEA, 40000 Marrakech, Morocco \\ e-mail: telhalkouj@yahoo.fr \\ 2 Université de Nice Sophia-Antipolis UMR 6525, Parc Valrose, 06108 Nice, France \\ ${ }^{3}$ École Normale Supérieure, route d'Essaouira, Laboratoire d'optique et optoélectronique, 4000 Marrakech, Morocco
}

Received 27 June 2007 / Accepted 27 September 2007

ABSTRACT

\begin{abstract}
Context. Extending the potential of multi-aperture telescopes toward the higher magnitudes necessary for extragalactic science needs an off-axis reference source to cophase the interferometric array.

Aims. The off-axis reference star and the science source must be within the isopistonic angle, and evaluating this parameter is crucial for estimating the potential of long-baseline interferometers for faint sources, differential astrometry, and phase-reference imaging. Methods. We derived an analytical method of deducing the isopistonic angle from the standard atmospheric optical parameters, and used a full description of the atmospheric turbulence to validate numerical integrations.

Results. We used the analytical expression for the isopistonic angle for predictions concerning the VLT, Keck, and LBT interferometers, as well as for an array of small apertures in the Antarctica site Dome $\mathrm{C}$, indicating that is might be a unique site on earth for interferometric observations of faint sources.
\end{abstract}

Key words. instrumentation: interferometers - atmospheric effects

\section{Introduction}

To reach a suitable limiting magnitude with a multi-aperture interferometer, we need to cophase the different telescopes using a reference source (Mariotti 1994). This source should be located in the same isopistonic domain as the science source, which means that the differential atmospheric piston, within certain specifications, is the same in the direction of the two stars.

Because of the lack of isopistonic measurements, many studies of the piston effect have been carried out (Perrin 1997); (Quirrenbach et al. 1994). Recently, Esposito derived a compact expression of the differential piston variance (Esposito et al. 2000). This was done with the spatial correlation of ZERNIKE modes of the wave front with a finite outer scale developed by Takato in (Takato et al. 1995). This expression requires those $C_{N}^{2}$ profiles that are not available in literatures and that need specific instruments for their measurements. Moreover, calculations require a numerical integration that is not always a simple task.

Using the MELLIN transform (Sasiela 1994), we developed an analytical expression for the differential piston variance depending on the accessed FRIED parameter $r_{0}$ instead of $C_{N}^{2}$ profiles. This expression enables us to establish a new formula for the isopistonic angle $\theta_{p}$ as an analytical function of the isoplanatic angle $\theta_{0}$, the FRIED parameter $r_{0}$, the atmospheric outer scale $L_{0}$, and the aperture diameter $D$. This formula is then used to fill the gap in isopistonic angle measurements. Indeed, using previous statistics for the isoplanatic angle, we are now able to provide new statistics for the isopistonic angle.

\section{Theoretical background}

To study only the effect of the differential piston on a twoaperture interferometer, we considered an ideal adaptive optics system. That lead us to suppose that the only effect of the atmosphere is a constant offset with different values on the portions of wavefronts on each of the two apertures, which is called the differential piston.

With the assumption of an ideal photometric calibration between the two arms and ignoring the diffraction effect of a single aperture, the fringe pattern on the image plane is given by (Goodman 2000)

$I=2 I_{0}\left[1+\operatorname{Re}\left(\gamma_{12}(\tau)\right)\right]$

where $\gamma_{12}(\tau)$ is the complex degree of coherence for a delay time $\tau$ between the two optical paths at the observation point in the interferometer image plane. Considering the instant visibility $\left.V=\mid \gamma_{12}(\tau)\right) \mid$, Eq. (1) can be written as

$I=2 I_{0}\left[1+V \cos \left(\varphi(\tau)-\varphi_{p}\right)\right]$

where $\varphi(\tau)$ is the phase due to the optical path difference between the arms, and $\varphi_{p}$ is the phase caused by the differential piston, with variance $\sigma_{\varphi, p}^{2}$. In the image plane, $\varphi(\tau)$ is constant, but there are a number of mechanisms (not described here) that lead to coherence losses and therefore to visibility reduction. Assuming a Gaussian behavior for $\varphi_{p}$, one can show that $V^{2}$ will be reduced by a factor $\mathrm{e}^{-\sigma_{\varphi, p}^{2}}$ or

$V_{\text {average }}=V \mathrm{e}^{-\frac{\sigma_{\varphi, p}^{2}}{2}}$. 


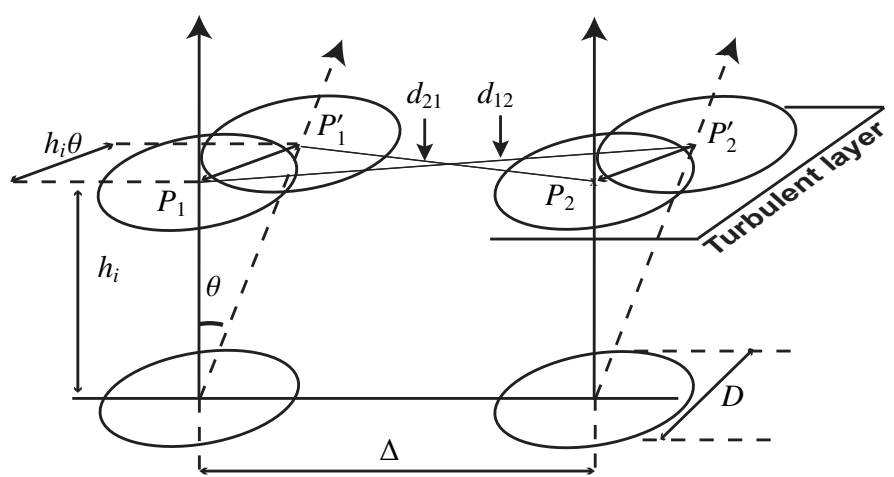

Fig. 1. Projected baseline and pupils according to two directions forming an angle $\theta$. The projection is made on a single turbulent layer with height $h$, (Esposito et al. 2000).

If we assume that the angular anisoplanatism is the only mechanism that leads to the visibility reduction, then

$V_{\text {average }}(\theta)=V \mathrm{e}^{-\frac{2 \pi^{2}}{\lambda^{2}} \sigma_{p}^{2}(\theta)}$

where $\sigma_{p}^{2}(\theta)$ is the isopistonic variance of the differential piston error (also called anisopistonic error variance).

Let now us consider a two-aperture interferometer pointing an off-axis target at an angular distance $\theta$ from the scientific target axis, and in the case of a single turbulent layer at an altitude $h$, let $P_{1}, P_{2}$ and $P_{1}^{\prime}, P_{2}^{\prime}$ be the pupil's projection onto this layer along the two pointed directions as shown in Fig. 1.

The expression of the isopistonic variance between $P_{1}, P_{2}$ and $P_{1}^{\prime}, P_{2}^{\prime}$ pairs of apertures, is given by (Esposito et al. 2000):

$$
\begin{aligned}
& \sigma_{p}^{2}(\theta)=\eta \pi^{2 / 3} \lambda^{2}\left(\frac{D}{r_{0, h_{i}}}\right)^{5 / 3} \int_{0}^{\infty} \frac{J_{1}^{2}(x)}{x\left[x^{2}+\left(\pi \frac{D}{L_{0, i}}\right)^{2}\right]^{\frac{11}{6}}} \\
& \times\left\{2\left[1-J_{0}\left(2 x \frac{h \theta}{D}\right)-J_{0}\left(2 x \frac{\Delta}{D}\right)\right]+J_{0}\left(2 x \frac{d_{12}}{D}\right)+J_{0}\left(2 x \frac{d_{21}}{D}\right)\right\} \mathrm{d} x
\end{aligned}
$$

where $\eta$ is a constant, $\eta=[(24 / 5) \Gamma(6 / 5)]^{5 / 6} \Gamma^{2}(11 / 6) / 2 \pi^{11 / 3}=$ 0.0229 (Conan et al. 2000), $D$ is the aperture diameter, $\Delta$ the baseline interferometer, $L_{0, i}$ the outer scale at altitude $h_{i}$; and $d_{12}$ (respectively $d_{21}$ ) the distance between the pupils $P_{1}$ and $P_{2}^{\prime}$ (respectively $P_{1}^{\prime}$ and $P_{2}$ ), with the following cases:

- $d_{12}=d_{21}=\sqrt{\Delta^{2}+\left(h_{i} \theta\right)^{2}}$, if the observation of the reference source is made orthognally to the baseline;

- $d_{12}=\Delta \pm h_{i} \theta$, and $d_{21}=\Delta \mp h_{i} \theta$, if the observation of the reference source is done along the direction of the baseline.

In the case of a single turbulent layer of thickness $\Delta h$ at altitude $h_{i}$, the expression of the FRIED parameter is given by (Fried 1966)

$\lambda^{2} r_{0, h_{i}}^{-\frac{5}{3}}=\frac{1}{0.06} C_{N}^{2}\left(h_{i}\right) \Delta h$.

Thus, the expression of $\sigma_{p}^{2}$ becomes

$$
\begin{aligned}
& \sigma_{p}^{2}(\theta)=1.5 \pi^{2 / 3} D^{5 / 3} C_{N}^{2}\left(h_{i}\right) \Delta h \int_{0}^{\infty} \frac{J_{1}^{2}(x)}{x\left[x^{2}+\left(\pi \frac{D}{L_{0}, i}\right)^{2}\right]^{\frac{11}{6}}} \\
& \times\left\{2\left[1-J_{0}\left(2 x \frac{h_{i} \theta}{D}\right)-J_{0}\left(2 x \frac{\Delta}{D}\right)\right]+J_{0}\left(2 x \frac{d_{12}}{D}\right)+J_{0}\left(2 x \frac{d_{21}}{D}\right)\right\} \mathrm{d} x .
\end{aligned}
$$

To obtain the isopistonic variance of the whole atmosphere, we consider an equivalent outer scale $L_{0}$. Using the hypothesis of independent turbulent layers (near field approximation), which means that the overall error variance will be the sum of the single-layer error variances, one can write

$$
\sigma_{p}^{2}(\theta)=\sum_{i} \sigma_{p}^{2}\left(\theta, h_{i}\right)
$$

by making the assumption of a continuous distribution of atmosphere, the isopistonic variance of the whole atmosphere is

$$
\begin{aligned}
\sigma_{p}^{2}(\theta)= & 1.5 \pi^{2 / 3} D^{5 / 3} \int_{0}^{\infty} \int_{0}^{\infty} C_{N}^{2}(h) \frac{J_{1}^{2}(x)}{x\left[x^{2}+\left(\pi \frac{D}{L_{0}}\right)^{2}\right]^{\frac{11}{6}}} \\
& \times\left\{2\left[1-J_{0}\left(2 x \frac{h \theta}{D}\right)-J_{0}\left(2 x \frac{\Delta}{D}\right)\right]\right. \\
& \left.+J_{0}\left(2 x \frac{d_{12}}{D}\right)+J_{0}\left(2 x \frac{d_{21}}{D}\right)\right\} \mathrm{d} x \mathrm{~d} h .
\end{aligned}
$$

This equation gives the total anisopistonic error variance. However, this expression is not practical, first of all because the scarcity of the $C_{N}^{2}$ profiles in the literature, then the need for specific instruments for its measurements, and finally because it requires a double numerical integration that is not always easy to achieve.

To obtain a simpler expression of the total anisopistonic variance, we begin by reducing the last equation in the case of large baseline interferometers. This is the object of the next section.

\section{Analytical expression of isopistonic angle variance}

Isoplanatic and isopistonic angles are dominated by highaltitude layers $(h>10 \mathrm{~km})$; and due to sky coverage and limiting magnitude, we should consider that $h$ and $\theta$ are large. However in the case of long baseline interferometers - KECK $(\Delta=80 \mathrm{~m}), \operatorname{VLTI}\left(\Delta_{\mathrm{UT} 1-\mathrm{UT} 2}=130 \mathrm{~m}\right), \operatorname{LBT}(\Delta=14.4 \mathrm{~m})$, etc. - the distance $h \theta$ is in general much smaller than the baseline $\Delta$ ( $h \theta \simeq 1 \mathrm{~m}$, for $h \simeq 20 \mathrm{~km}$ and $\theta \simeq 10 \mathrm{arsec}$ ). Then, we can make the following approximation $\Delta \simeq d_{12} \simeq d_{21}$, so we can simplify Eq. (6):

$$
\begin{aligned}
\sigma_{p}^{2}(\theta)= & 3 \pi^{2 / 3} D^{5 / 3} \int_{0}^{\infty} \int_{0}^{\infty} \frac{J_{1}^{2}(x) C_{N}^{2}(h)}{x\left[x^{2}+\left(\pi \frac{D}{L_{0}}\right)^{2}\right]^{\frac{11}{6}}} \\
& \times\left[1-J_{0}\left(2 x \frac{h \theta}{D}\right)\right] \mathrm{d} x \mathrm{~d} h .
\end{aligned}
$$

To verify the validity of this approximation, we calculated the two integrals in Eqs. (6) and (7) numerically for various values of $\theta$ and of the outer scale $L_{0}$. We restricted this verification to the unfavourable case, i.e., the shortest baseline corresponding to the LBT interferometer. The $C_{N}^{2}(h)$ profile used in numerical computation is the Hufnagel-Valley model (Beckers 1993), normalized to $r_{0}(0.55 \mu \mathrm{m})=13.3 \mathrm{~cm}$.

We plotted in Fig. 2 the relative difference between the results of Eqs. (6) and (7) for different values of $\theta$ :

$E_{r}=\frac{\left|\sigma_{p, \text { Eq. (7) }}^{2}(\theta)-\sigma_{p, \text { Eq. (6) }}^{2}(\theta)\right|}{\sigma_{p, \text { Eq. (6) }}^{2}(\theta)}$. 


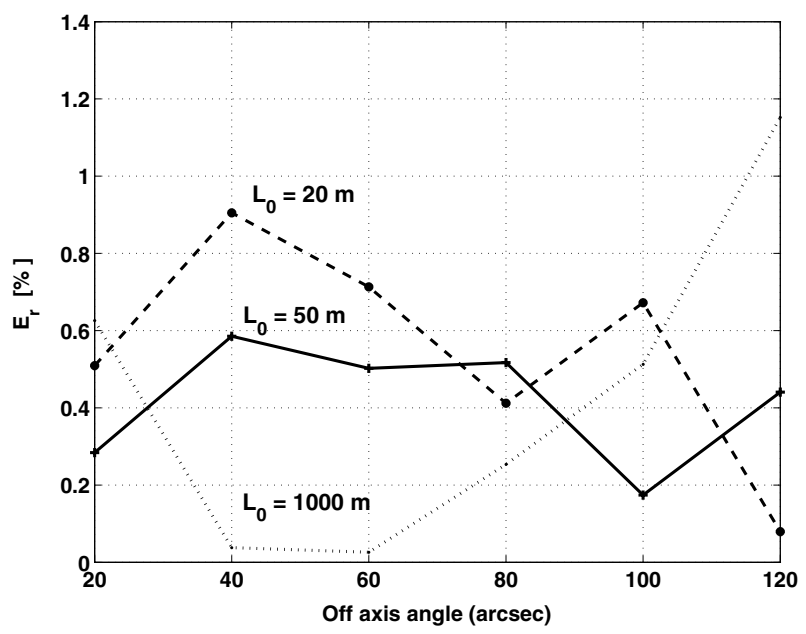

Fig. 2. Curves representing the variations in the relative difference (in \%) between Eqs. (7) and (6). As indicated in the figure, these curves are performed for three values 20,50 , and $1000 \mathrm{~m}$ on the outer scale $L_{0}$.

As shown in Fig. 2, the relative difference is less than $2 \%$, confirming the validity of this long-baseline approximation.

In order to obtain an analytical expression for the integral Eq. (7), we first rewrite it in a compact form using the dimensionless variables $a=\frac{\pi D}{L_{0}}$ and $b=\frac{D}{2 h \theta}$ :

$\sigma_{p}^{2}(\theta)=3 \pi^{2 / 3} D^{5 / 3} \int_{0}^{\infty} C_{N}^{2}(h)\left[I_{0}(a)-I_{1}(a, b)\right] \mathrm{d} h$

where

$I_{0}(a)=\int_{0}^{\infty} \frac{J_{1}^{2}(x)}{x\left[x^{2}+a^{2}\right]^{\frac{11}{6}}} \mathrm{~d} x=a^{-\frac{11}{3}} \int_{0}^{\infty} \frac{J_{1}^{2}(x)}{\left[1+\left(\frac{a}{x}\right)^{-2}\right]^{\frac{11}{6}}} \frac{\mathrm{d} x}{x}$

and

$I_{1}(a, b)=\int_{0}^{\infty} \frac{J_{1}^{2}(x) J_{0}\left(\frac{x}{b}\right)}{x\left[x^{2}+a^{2}\right]^{\frac{11}{6}}} \mathrm{~d} x=a^{-\frac{11}{3}} \int_{0}^{\infty} \frac{J_{1}^{2}(x) J_{0}\left(\frac{x}{b}\right)}{\left[1+\left(\frac{a}{x}\right)^{-2}\right]^{\frac{11}{6}}} \frac{\mathrm{d} x}{x}$.

These integrals can be expressed as convergent series using a technique developed by Sasiela (1994). This technique is based on the convolution theorem of the MELLIN transform. The calculations gathered in the appendix show that the choice of convergent series depends on the parameters $a$ and $b$. Indeed the integral $I_{0}(a)$ is expressed in terms of TAYLOR or asymptotic series depending on parameter $a$ :

- $a<1$.

In this range, the integral $I_{0}(a)$ can be developed as TAYLOR series (T) (Sasiela 1994)

$I_{0}(a)=\frac{a^{\frac{-11}{3}}}{2 \sqrt{\pi} \Gamma\left(\frac{11}{6}\right)} \sum_{n=0}^{\infty} \frac{(-1)^{n}}{n !}\left(\alpha_{0 n}^{\mathrm{T}} a^{2 n+2}+\beta_{0 n}^{\mathrm{T}} a^{2 n+\frac{11}{3}}\right)$

where

$\alpha_{0 n}^{\mathrm{T}}=\frac{\Gamma[3 / 2+n, 1+n, 5 / 6-n]}{\Gamma[3+n, 2+n]}$

$\beta_{0 n}^{\mathrm{T}}=\frac{\Gamma[-n-5 / 6,7 / 3+n, 11 / 6+n]}{\Gamma[n+23 / 6, n+17 / 6]}$

with $\quad \Gamma[x, y, z]=\Gamma(x) \Gamma(y) \Gamma(z)$.

Gamma function $\Gamma(x)$ is defined by

$\Gamma(s)=\int_{0}^{\infty} \mathrm{d} x \exp (-x) x^{s-1}, \quad s \in \mathbb{C}$

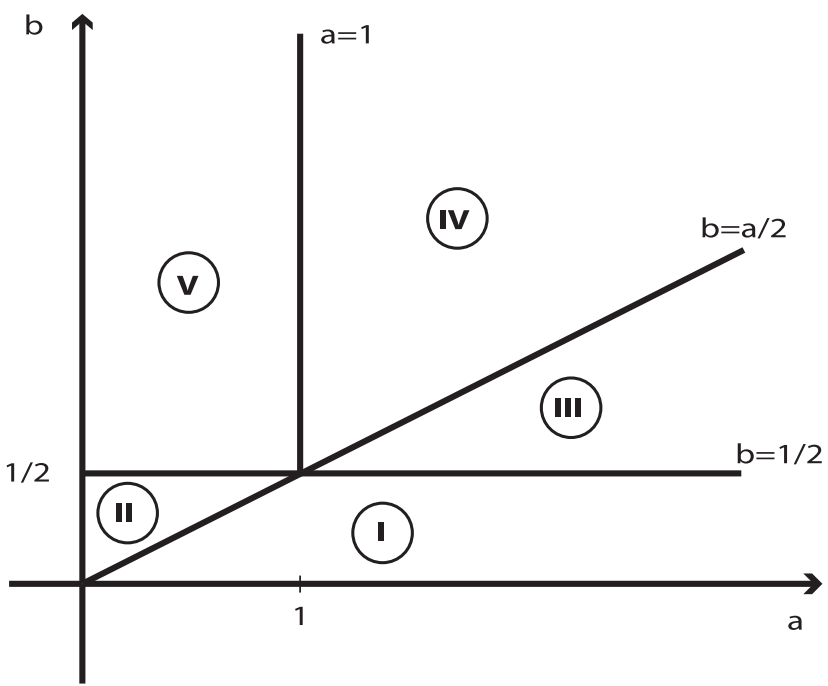

Fig. 3. Domains in the $(a, b)$ plane that are required by the technique of MELLIN transform. (I): $b<1 / 2$ and $b<a / 2$; (II): $a / 2<b<1 / 2$; (III): $1 / 2<b<a / 2$; (IV): $b>a / 2$ and $a>1 ;(\mathbf{V}): b>1 / 2$ and $a<1$.

- $a>1$.

In this case, the integral $I_{0}(a)$ could be expressed as an asymptotic series (A):

$I_{0}(a)=\frac{a^{\frac{-11}{3}}}{2 \sqrt{\pi} \Gamma\left(\frac{11}{6}\right)} \sum_{n=0}^{\infty} \frac{(-1)^{n}}{n !}\left(\alpha_{0 n}^{\mathrm{A}} a^{-2 n-1}+\beta_{0 n}^{\mathrm{A}} a^{-2 n}\right)$

where

$\alpha_{0 n}^{\mathrm{A}}=\frac{\Gamma[3 / 2+n,-1 / 2-n, 7 / 3+n]}{\Gamma[3 / 2-n, 1 / 2-n]}$

$\beta_{0 n}^{\mathrm{A}}=\frac{\Gamma[1+n, 1 / 2-n, 11 / 6+n]}{\Gamma[2-n, 1-n]}$.

One can notice that $\beta_{0 n}^{(\mathrm{A})}=0$ for $n \neq 0$.

From Table 5 in the Appendix, we can distinguish for the integral $I_{1}(a, b)$ five domains in the $(a, b)$ plane, which are represented in Fig. 3:

- domain (I): $b<1 / 2$ and $b<a / 2$;

- domain (II): $a / 2<b<1 / 2$;

- domain (III): $1 / 2<b<a / 2$;

- domain (IV): $b>a / 2$ and $a>1$;

- domain (V): $b>1 / 2$ and $a<1$.

Domains (I) and (III) are not considered since they require that $\left(L_{0} / \pi\right)<(h \theta)_{\min }$, with $(h \theta)_{\min } \simeq 0$. For the same reason, domain (II) requires that $D<(h \theta)_{\min }$, which makes no sense. The remaining domains (IV) and (V) correspond respectively to large and small apertures compared to the outer scale value.

- For the small-aperture case (V: $b>1 / 2$ and $a<1)$, the calculations from the Appendix show that the integral $I_{1}(a, b)$ can be expressed as

$$
\begin{aligned}
I_{1}(a, b)= & \frac{a^{\frac{-11}{3}}}{2 \sqrt{\pi} \Gamma\left(\frac{11}{6}\right)} \sum_{n=0}^{\infty} \sum_{m=0}^{\infty} \frac{(-1)^{n}}{n !} \frac{(-1)^{m}}{m !} \\
& \times\left[\alpha_{1 n m}^{\mathrm{T}} a^{2 n+2}\left(\frac{a}{2 b}\right)^{2 m}+\beta_{1 n m}^{\mathrm{T}}(2 b)^{-2 n-1}\left(\frac{a}{2 b}\right)^{2 m+\frac{11}{3}}\right. \\
& \left.+\gamma_{1 n m}^{\mathrm{T}} a^{2 n+\frac{11}{3}}(2 b)^{-2 m}\right]
\end{aligned}
$$


with

$$
\begin{aligned}
\alpha_{1 n m}^{\mathrm{T}} & =\frac{\Gamma[3 / 2+n, 1+n+m, 5 / 6-m-n]}{\Gamma[3+n, 2+n, 1+m]} \\
\beta_{1 n m}^{\mathrm{T}} & =\frac{\Gamma[3 / 2+n, m+11 / 6,-7 / 3-m-n]}{\Gamma[3 / 2-n, 1 / 2-n, 10 / 3+m+n]} \\
\gamma_{1 n m}^{\mathrm{T}} & =\frac{\Gamma[m-n-5 / 6,7 / 3+n-m, 11 / 6+n]}{\Gamma[23 / 6+n-m, 17 / 6+n-m, 1+m]} .
\end{aligned}
$$

- In the case of large-aperture (IV: $b>a / 2$ and $a>1$ ), the development of the integral $I_{1}(a, b)$ leads to (see the Appendix)

$$
\begin{aligned}
I_{1}(a, b)= & \frac{a^{\frac{-11}{3}}}{2 \sqrt{\pi} \Gamma\left(\frac{11}{6}\right)} \sum_{n=0}^{\infty} \sum_{m=0}^{\infty} \frac{(-1)^{n}}{n !} \frac{(-1)^{m}}{m !} \\
& \times\left[\begin{array}{r}
\alpha_{1 n m}^{\mathrm{A}} a^{-2 n-1}\left(\frac{a}{2 b}\right)^{2 m}+\beta_{1 n m}^{\mathrm{A}} a^{-2 n}(2 b)^{-2 m} \\
\\
+\gamma_{1 n m}^{\mathrm{T}}\left(\frac{a}{2 b}\right)^{2 m+\frac{11}{3}}(2 b)^{-2 n-1}
\end{array}\right]
\end{aligned}
$$

with

$$
\begin{aligned}
\alpha_{1 n m}^{\mathrm{A}} & =\frac{\Gamma[3 / 2+n,-1 / 2-n+m, 7 / 3+n-m]}{\Gamma[3 / 2-n, 1 / 2-n, 1+m]} \\
\beta_{1 n m}^{\mathrm{A}} & =\frac{\Gamma[1+n+m, 1 / 2-n-m, n+11 / 6]}{\Gamma[2-n-m, 1-n-m, 1+m]} \\
\gamma_{1 n m}^{\mathrm{T}} & =\frac{\Gamma[3 / 2+n, m+11 / 6,-7 / 3-n-m]}{\Gamma[3 / 2-n, 1 / 2-n, 10 / 3+n+m]} .
\end{aligned}
$$

Using these developments, the difference in integrals $I_{0}(a)$ $I_{1}(a, b)$, which we shall call $I(a, b)$, is given by

$$
\begin{aligned}
I(a, b) & =\sum_{i} P_{i}(a)\left(\frac{1}{b}\right)^{2 i}+\sum_{j} P_{2 j+14 / 3}(a)\left(\frac{1}{b}\right)^{2 j+14 / 3} \\
& =\sum_{k} P_{k}(a) \frac{1}{b^{k}}
\end{aligned}
$$

where $P_{k}(a)$ is a polynomial of the parameter a and $k=2 i$ or $k=2 j+14 / 3$.

Combining Eqs. (13) and (8) and replacing $b$ by its expression $b=D / 2 h \theta$ leads to the following expression of the anisopistonic variance:

$$
\begin{aligned}
\sigma_{p}^{2}(\theta)= & 3 \pi^{2 / 3} D^{5 / 3} \int_{0}^{\infty} C_{N}^{2}(h) \mathrm{d} h \\
& \times\left[\sum_{k} P_{k}(a)\left(\frac{2}{D}\right)^{k} \theta^{k} \frac{\int_{0}^{\infty} h^{k} C_{N}^{2}(h) \mathrm{d} h}{\int_{0}^{\infty} C_{N}^{2}(h) \mathrm{d} h}\right] .
\end{aligned}
$$

Introducing the $n$th turbulence altitude moment,

$h_{n}=\left[\frac{\int_{0}^{\infty} h^{n} C_{N}^{2}(h) \mathrm{d} h}{\int_{0}^{\infty} C_{N}^{2}(h) \mathrm{d} h}\right]^{1 / n}$,

and using the $r_{0}$ expression deduced from Eq. (5) generalized to the whole atmosphere, we can write

$\sigma_{p}^{2}(\theta)=0.18 \pi^{2 / 3} \lambda^{2}\left(\frac{D}{r_{0}}\right)^{\frac{5}{3}} \sum_{k} P_{k}(a)\left(\frac{2}{D}\right)^{k}\left(h_{k}\right)^{k} \theta^{k}$.

For $n=5 / 3$, the expression of $h_{5 / 3}$ is related (Roddier et al. 1982) to the FRIED parameter $r_{0}$ and the isoplanatic angle $\theta_{0}$ by: $h_{5 / 3}=0.31 \frac{r_{0}}{\theta_{0}}$.
Assuming that $h_{k}=\alpha(k) h_{5 / 3}$, where $\alpha(k)$ is a correcting term that can be evaluated from numerical computation of the $C_{N}^{2}(h)$ profile, we evaluated this correcting term for several sites using the Hufnagel-Valley model for LBT (Beckers 1993), the $C_{N}^{2}$ profiles measurements above Mauna Kea (Tokovinin et al. 2005), and Cerro Paranal (Sarazin 1995; Flight 48).

For computational purpose, it is convenient to replace $\alpha(k)$ by an analytical function obtained by fitting the numerical values of $\alpha(k)$. The following expressions provide an excellent fit for the correcting term:

$\begin{array}{ll}\text { LBT } & \alpha(k)=2.6 \times 10^{-4} k^{3}-0.011 k^{2}+0.24 k+0.69 \\ \text { KECK } & \alpha(k)=2.9 \times 10^{-4} k^{3}-0.014 k^{2}+0.27 k+0.61 \\ \text { VLTI } & \alpha(k)=8.2 \times 10^{-4} k^{3}-0.037 k^{2}+0.62 k+0.09\end{array}$

This leads to the general analytical expression:

$\sigma_{p}^{2}(\theta)=0.18 \pi^{2 / 3} \lambda^{2}\left(\frac{D}{r_{0}}\right)^{\frac{5}{3}} \sum_{k}\left(\frac{0.62 r_{0}}{D}\right)^{k}[\alpha(k)]^{k} P_{k}(a)\left(\frac{\theta}{\theta_{0}}\right)^{k}$

where $\sigma_{p}^{2}(\theta)$ is now a polynomial of $\theta$. Moreover, Eq. (17) allows us to directly obtain the $\sigma_{p}^{2}$ values, knowing one of the atmospheric and instrumental parameters $\theta_{0}, r_{0}, L_{0}$, and $D$. Finally this analytical expression of $\sigma_{p}^{2}$ leads to the isopistonic angle, while Eq. (7) requires a numerical resolution to get each value of the isopistonic angle. In the following we give a simpler expression of the isopistonic variance by limiting the development.

To avoid the effect of $C_{N}^{2}$ weighting on the $\sigma_{p}^{2}$, we first evaluate the error made in the approximation of the $I(a, b)$ integral difference. In the cases of small and large apertures, we compared series developments in Eq. (13) and numerical integration: $I(a, b)_{\text {integ }}=I_{0}(a)-I_{1}(a, b)$. At the same time, we checked that, without losing more accuracy, we can limit the development in Eq. (13) to the fourth order $(k=0,2,4)$. Moreover, we checked that, for $k=0$, the polynomial $P_{0}(a)$ is practically zero for all values of parameter $a$. Therefore the series development $I(a, b)_{\text {series }}$ used is

$I(a, b)_{\mathrm{series}}=P_{2}(a) \frac{1}{b^{2}}+P_{4}(a) \frac{1}{b^{4}}$.

We note that $\alpha(2) \simeq 1.13$ for the all previous sites (by a relative difference $\leq 5 \%$ ), which is expected because $5 / 3 \simeq 2$, while the term $\alpha(4)$ varies with the site: $\alpha(4)_{\mathrm{LBT}} \simeq 1.51, \alpha(4)_{\mathrm{KECK}} \simeq 1.44$, and $\alpha(4)_{\mathrm{VLTI}} \simeq 2.14$. With these considerations in mind, Eq. (17) becomes

$$
\begin{aligned}
& \sigma_{p}^{2}(\theta)=6.9 \times 10^{-2} \pi^{2 / 3} \lambda^{2}\left(\frac{D}{r_{0}}\right)^{-\frac{1}{3}} \\
& \times\left[1.27 P_{2}(a)+0.4[\alpha(4)]^{4}\left(\frac{r_{0}}{D}\right)^{2} P_{4}(a)\left(\frac{\theta}{\theta_{0}}\right)^{2}\right]\left(\frac{\theta}{\theta_{0}}\right)^{2} .
\end{aligned}
$$

This general expression of $\sigma_{p}^{2}(\theta)$ still depends on the $C_{N}^{2}$ profiles via the correcting term $\alpha(4)$, and we show in the following that it will no be longer the case under certain considerations.

\subsection{Small-aperture case}

Considering $b>1 / 2$ and $a<1$, we have plotted in Fig. 4 the relative difference $\epsilon(a, b)$ between the integral calculated by series expansion and the one calculated by numerical computation:

$\epsilon(a, b)=\frac{\left|I(a, b)_{\text {integ }}-I(a, b)_{\text {series }}\right|}{I(a, b)_{\text {integ }}}$ 


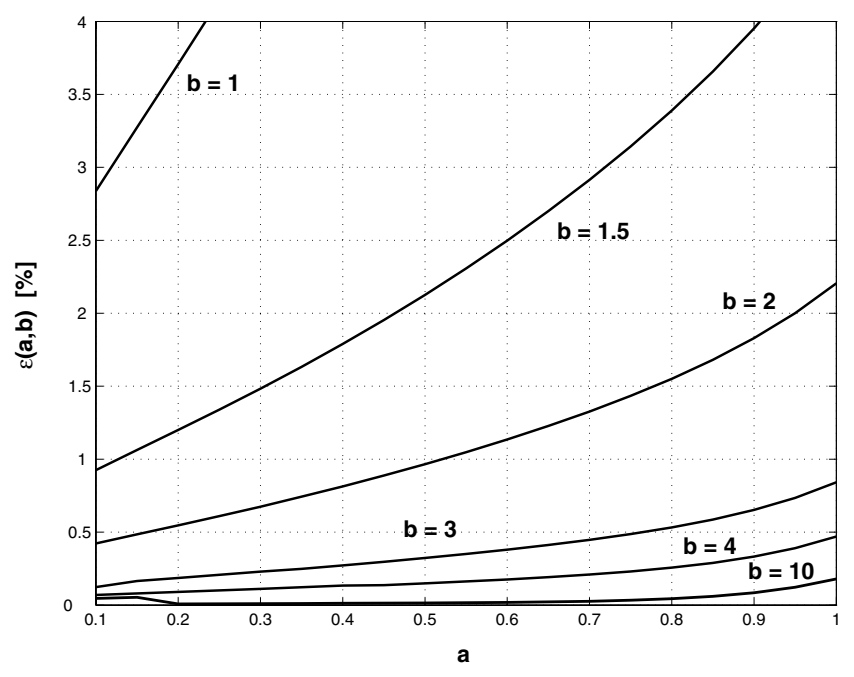

Fig. 4. Relative difference between the results obtained by numerical computation of the integral expression $I_{0}(a)-I_{1}(a, b)$ and its series expansion with the corresponding values $1,1.5,2,3,4$, and 10 of parameter $b$.

where $I(a, b)_{\text {series }}$ is calculated using Eqs. (9) and (11). This leads to $P_{2}(a)$ and $P_{4}(a)$ :

$$
\begin{aligned}
P_{2}(a)= & 0.216-0.225 a^{1 / 3}+0.122 a^{2}-0.096 a^{7 / 3}+0.02 a^{4} \\
& -0.014 a^{13 / 3}+1.64 \times 10^{-3} a^{6}-10^{-3} a^{19 / 3}
\end{aligned}
$$

and

$$
\begin{aligned}
P_{4}(a)= & -0.01+2.47 \times 10^{-2} a^{2}-2.41 \times 10^{-2} a^{7 / 3}+0.01 a^{4} \\
& -8.34 \times 10^{-3} a^{13 / 3}+1.61 \times 10^{-3} a^{6}-1.1 \times 10^{-3} a^{19 / 3} .(22)
\end{aligned}
$$

According to Fig. 4, we note that the relative difference increases with parameter $a$ and decreases with parameter $b$. The approximation is less valid when diameter $D$ increases and /or outer scale $L_{0}$ decreases. We note finally that the small-aperture case can be extended to $a \leq 1.3$; i.e $D \leq 1.3 L_{0} / \pi$, which will be verified at the end of this section.

Using the expressions of $P_{2}(a)$, Eq. (21), and $P_{4}(a)$, Eq. (22) in Eq. (19) gives the general expression of isopistonic variance in the small-aperture case. Since the aperture diameter of most known interferometers is large, Eq. (19) is reduced to a simpler one. Indeed, we have verified that, for $b>1.5$, i.e $D>3 h \theta$, the first term on the right-hand side of Eq. (18) gives the same error as in Fig. 4, which means that $I(a, b)_{\text {series }}=P_{2}(a) / b^{2}$. In this case and assuming that $D>3(h \theta)_{\max }$, Eq. (19) is reduced to the tractable Eq. (23).

In order to evaluate the parameter $(h \theta)_{\max }$, we assume it equal to $h_{\max } \theta_{0}$ where $h_{\max }$ is the highest altitude with significant turbulence energy, $\left(h_{\max } \sim 20 \mathrm{~km}\right.$ for almost all sites) and $\theta_{0}$ is the isoplanatic angle at the same site.

The isopistonic variance expression is now

$$
\begin{aligned}
& \sigma_{p}^{2}(\theta)=8.8 \times 10^{-2} \pi^{2 / 3} \lambda^{2}\left(\frac{D}{r_{0}}\right)^{-\frac{1}{3}}\left(\frac{\theta}{\theta_{0}}\right)^{2} \\
& \times\left[0.216-0.225\left(\frac{\pi D}{L_{0}}\right)^{1 / 3}+0.122\left(\frac{\pi D}{L_{0}}\right)^{2}-0.096\left(\frac{\pi D}{L_{0}}\right)^{7 / 3}\right. \\
& \left.+0.02\left(\frac{\pi D}{L_{0}}\right)^{4}-0.014\left(\frac{\pi D}{L_{0}}\right)^{13 / 3}\right], \text { with }\left\{\begin{array}{c}
D \leq 1.3 \frac{L_{0}}{\pi} \\
D \geq 3 h_{\max } \theta_{0} .
\end{array}\right.
\end{aligned}
$$

We notice that the expression of $\sigma_{p}^{2}(\theta)$, Eq. (23), is independent of the $C_{N}^{2}$ profiles.

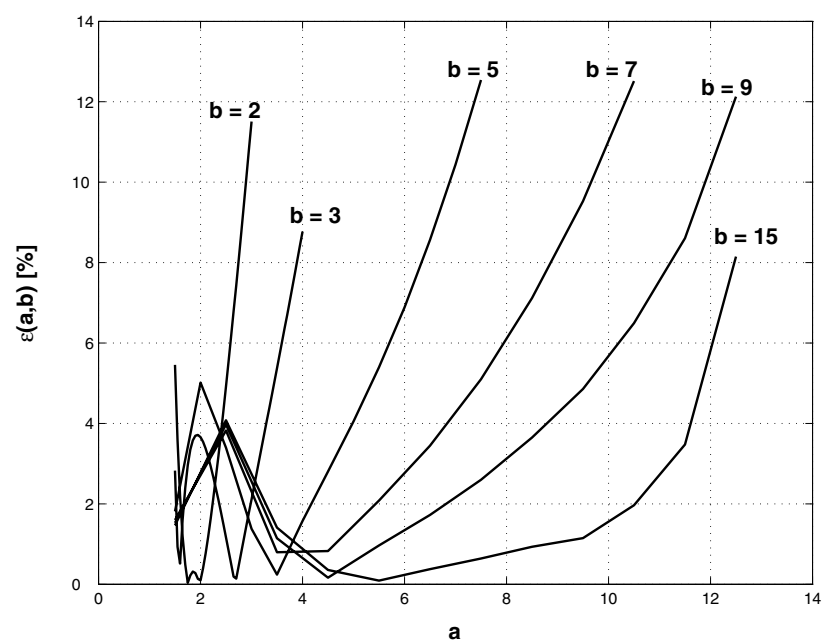

Fig. 5. Relative difference between the results obtained from numerical computation of the integral expression $I_{0}(a)-I_{1}(a, b)$ and those obtained from the series expansion with the corresponding values $2,3,5,7,9$, and 15 of the parameter $b$.

When the outer scale values are very high (KoLMOGoRov model), the parameter $a=\pi D / L_{0}$ is very small compared to unity. From Eq. (23) we obtain the isopistonic angle error:

$\sigma_{p}(\theta) \sim 0.12 \pi^{1 / 3} \lambda\left(\frac{D}{r_{0}}\right)^{-1 / 6} \frac{\theta}{\theta_{0}} \quad L_{0} \rightarrow \infty$.

\subsection{Large-aperture case}

In this case, the polynomials $P_{2}(a)$ and $P_{4}(a)$ in Eq. (18) can be derived respectively by Eqs. (10) and (12) :

$P_{2}(a)=6.69 \times 10^{-2}\left(a^{-8 / 3}-a^{-14 / 3}\right)$

and

$P_{4}(a)=-1.57 \times 10^{-3} a^{-8 / 3}-6.28 \times 10^{-3} a^{-14 / 3}$.

We have plotted in Fig. 5 the relative difference $\epsilon(a, b)$ Eq. (20) as a function of the parameters $a$ and $b$. One can note that the relative error decreases when $b$ increases, and the domains of good accuracy depend on both the parameters $a$ and $b$. Moreover, the $\epsilon(a, b)$ values are high enough compared to small-aperture ones. This is due to asymptotic developments of integrals that converge slowly for low values of the parameters $a$ and $b$. Therefore to obtain an acceptable accuracy, we use the new domains indicated in Table 1 instead of the domain $1<a$ and $a<2 b$, which define the large-aperture case.

By using the same reasoning as in the previous section, the isopistonic angle variance for the large-aperture case is given by the general Eq. (19) in which $P_{2}(a)$ and $P_{4}(a)$ are given respectively by Eqs. (25) and (26). In addition for $D \geq 6 h_{\max } \theta_{0}$, the isopistonic variance can be expressed by Eq. (27) which is also independent of the $C_{N}^{2}$ profiles

$$
\begin{aligned}
\sigma_{p}^{2}(\theta)= & 5.9 \times 10^{-3} \pi^{2 / 3} \lambda^{2}\left(\frac{D}{r_{0}}\right)^{-\frac{1}{3}}\left(\frac{\theta}{\theta_{0}}\right)^{2} \\
& \times\left[\left(\frac{\pi D}{L_{0}}\right)^{-8 / 3}-\left(\frac{\pi D}{L_{0}}\right)^{-14 / 3}\right], \text { with }\left\{\begin{array}{c}
D>1.3 \frac{L_{0}}{\pi} \\
D \geq 6 h_{\max } \theta_{0} .
\end{array}\right.
\end{aligned}
$$


Table 1. Parameter ranges of applicability for the 4th degree polynomial $I(a, b)$ in $1 / b$.

\begin{tabular}{ccc}
\hline \hline Parameter & $b$ & $a$ \\
\hline Range & $2 \leq b \leq 7$ & $1.3<a<2 b-2$ \\
Range & $b>7$ & $1.4<a<13$ \\
\hline
\end{tabular}

Table 2. The atmospheric parameters and the single-aperture diameters for KECK, LBT, and VLTI used in the computation of anisopistonic error.

\begin{tabular}{lccc}
\hline \hline Interferometer & Diam. $(\mathrm{m})$ & $r_{0}(\mathrm{~cm})$ & $\theta_{0}(\operatorname{arcsec})$ \\
\hline VLTI (UT1-UT2) & 8.2 & 13.7 & 2.5 \\
Keck & 10 & 23.6 & 1.9 \\
LBT & 8.4 & 13.3 & 1.4 \\
\hline
\end{tabular}

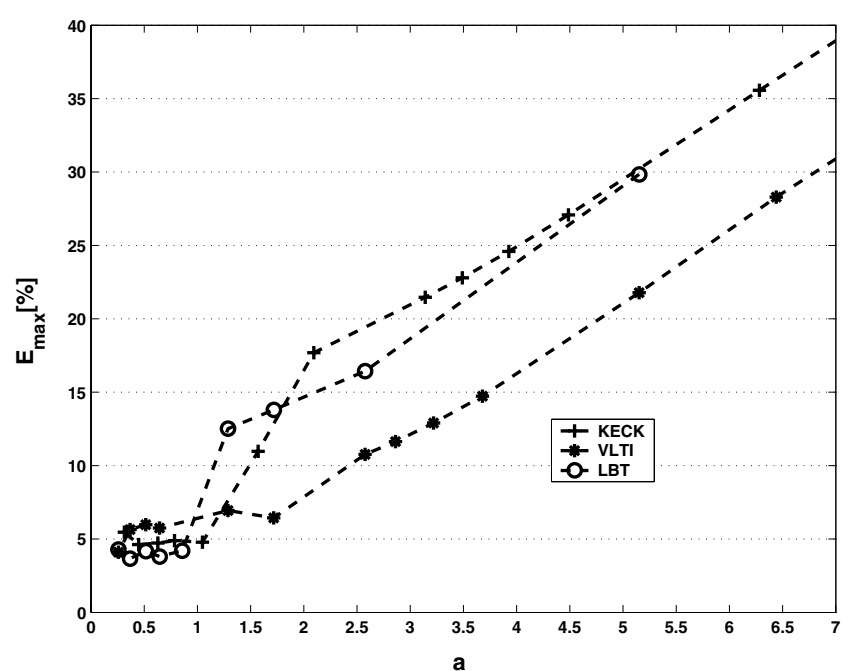

Fig. 6. Maximum of relative difference between the polynomial and integral forms of the anisopistonic error, at KECK, VLTI, and LBT.

\subsection{Application to some astronomical interferometers}

In this section we check the validity of the analytical expression of $\sigma_{p}^{2}$ with the conditions of the most-known interferometers KECK, LBT, and VLTI interferometers. Using parameter values given in Table 2, the Fig. 6 gives for KECK, LBT, and VLTI the respective maximum relative error $E_{\max }(a)$ defined as

$E_{\max }(a)=\operatorname{Max}_{/ \theta}\left(\frac{\left|\sigma_{p, \text { Eq. (6) }}(\theta)-\sigma_{p, \text { Eq. (19) }}(\theta)\right|}{\sigma_{p \text {, Eq. (6) }}(\theta)}\right)$,

where $a=\pi D / L_{0}$.

For numerical computation, we used for KECK and VLTI available SCIDAR measurements of the atmospheric turbulence profiles above Mauna Kea (Tokovinin et al. 2005) and Cerro Paranal (Sarazin 1995; Flight 48). For LBT we used the Hufnagel-Valley model (Beckers 1993).

Note: parameters $r_{0}$ and $\theta_{0}$ are provided from the $C_{N}^{2}$ profiles (KECK, VLTI) and from the Hufnagel model (LBT).

Figure 6 gives a consequent relative error for the largeaperture case, and this is predicted by Fig. 5, for which the errors are accumulated by integration over height $h$. These accumulations also affect the case of the small aperture: the error is typically $0.5 \%$ in Fig. 4 and about $5 \%$ in Fig. 6.

We have verified that the analytical expression of $\sigma_{p}^{2}(\theta)$ for the small-aperture case can be extended to the large-aperture case with good accuracy until $a \leq 1.3$, i.e $D \leq 1.3 L_{0} / \pi$. This has already been applied in Fig. 6 and given a maximum relative error of about $6 \%$. As we show in Sect. 4.2, the inequality $D>6 h_{\max } \theta_{0}$ is verified for the considered interferometers. We checked that Eq. (23) (small-aperture) and Eq. (27) (largeaperture) give the same relative difference as in Fig. 6.

In addition for the advantages cited above, the analytical expression of isopistonic variance is very practical for global study of sky coverage. This last combines a study of the fringe tracking limiting magnitude, the isopistonic angle within which the reference star must be found, and the star density in different sky directions.

\section{Isopistonic angle}

\subsection{Isopistonic versus isoplanatic angle}

The isopistonic angle $\theta_{p}$ is defined as the angular radius of a circular region where the anisopitonic error reduces the visibility in the image plane to no more than $80 \%$ of the unperturbed value (Esposito et al. 2000). From Eq. (4), this correspond to a residual piston error $\sigma_{p}$ about $\lambda / 10$. We feel that it is more convenient to adopt the following definition for the isopistonic angle $\theta_{p}$. The isopistonic angle $\theta=\theta_{p}$ is reached when

$\sigma_{p}\left(\theta_{p}\right)=\frac{\lambda}{n}$

where $\frac{\lambda}{n}$ is the desired accuracy.

Using criterion Eq. (29) and the $\sigma_{p}^{2}(a, \theta)$ expression given by Eq. (19), we provide the general expression of isopistonic angle

$\theta_{p} \simeq \frac{\theta_{0}}{\sqrt{0.3[\alpha(4)]^{4}\left|P_{4}(a)\right|}}\left(\frac{D}{r_{0}}\right)$

$\times \sqrt{0.49 P_{2}(a)-\sqrt{0.24\left[P_{2}(a)\right]^{2}+\frac{3.15[\alpha(4)]^{4}}{\pi^{2 / 3} n^{2}} P_{4}(a)\left(\frac{r_{0}}{D}\right)^{5 / 3}}}$

where $P_{2}(a)$ and $P_{4}(a)$ are given by Eqs. (21) and (22), respectively for the small-aperture case, and by Eqs. (25) and (26) for the large-aperture case. The correcting term $\alpha(4)$ is evaluated by using the $C_{N}^{2}$ profile for the considered site.

For known interferometers and future ELT, the singleaperture diameter is large enough to verify $D>3 h_{\max } \theta_{0}$ (smallaperture) and $D>6 h_{\max } \theta_{0}$ (large-aperture). This leads to the simpler expressions of isopistonic angle at $\lambda / n$ :

- Small-aperture case: $D \leq 1.3 L_{0} / \pi$ and $D \geq 3 h_{\max } \theta_{0}$

$$
\begin{aligned}
& \theta_{p}=\frac{2.3}{n} \theta_{0}\left(\frac{D}{r_{0}}\right)^{1 / 6}\left[0.216-0.225\left(\frac{\pi D}{L_{0}}\right)^{1 / 3}+0.122\left(\frac{\pi D}{L_{0}}\right)^{2}\right. \\
& \left.-0.096\left(\frac{\pi D}{L_{0}}\right)^{7 / 3}+0.02\left(\frac{\pi D}{L_{0}}\right)^{4}-0.014\left(\frac{\pi D}{L_{0}}\right)^{13 / 3}\right]^{-1 / 2} .
\end{aligned}
$$

From Fig. 6, the maximum of the relative error for isopistonic angle values provided from Eq. (31) is $E_{\max }(a) \simeq 5 \%$. - Large-aperture: $D>1.3 L_{0} / \pi$ and $D \geq 6 h_{\max } \theta_{0}$

$$
\theta_{p}=\frac{8.9}{n} \theta_{0}\left(\frac{D}{r_{0}}\right)^{1 / 6}\left[\left(\frac{\pi D}{L_{0}}\right)^{-8 / 3}-\left(\frac{\pi D}{L_{0}}\right)^{-14 / 3}\right]^{-1 / 2} \text {. }
$$

In this case, the maximum of relative error $E_{\max }$ increases with the ratio $a=\pi D / L_{0}$. From Fig. 6 it varies roughly as $E_{\max }(a) \simeq 3 a+3[\%]$. 
Table 3. Isopistonic angle values in $K$ band and for $\lambda / 10$ accuracy, at KECK, LBT, and VLTI interferometers.

\begin{tabular}{cccc}
\hline \hline$L_{0}(\mathrm{~m})$ & $\theta_{p, \text { KECK }}(\operatorname{arcsec})$ & $\theta_{p, \mathrm{LBT}}(\operatorname{arcsec})$ & $\theta_{p, \mathrm{VLTI}}(\operatorname{arcsec})$ \\
\hline 10 & 127.6 & 75.9 & 133.6 \\
15 & 80.2 & 49.7 & 88.2 \\
25 & 58.9 & 33.6 & 59.9 \\
50 & 33.9 & 22.9 & 41.1 \\
100 & 25.9 & 18.1 & 32.5 \\
\hline
\end{tabular}

Table 4. Isopistonic angle at Dome $\mathrm{C}$ in $K$ band and for for $\lambda / 10$ accuracy.

\begin{tabular}{cccl}
\hline \hline \multicolumn{4}{c}{ Isopistonic angle (arcsec) } \\
\hline$L_{0}(\mathrm{~m})$ & 5 & 10 & 20 \\
$D=1 \mathrm{~m}$ & 49.2 & 37.7 & 31.8 \\
$D=2 \mathrm{~m}$ & 85.9 & 55.3 & 42.3 \\
$D=3 \mathrm{~m}$ & 126.1 & 74.2 & 52.1 \\
\hline
\end{tabular}

\subsection{Application to some astronomical interferometers}

Using the parameters in Table 2, we compare first the parameter $h_{\max } \theta_{0}$ to the aperture diameter $D$ of the KECK, LBT, and VLTI. Assuming $h_{\max }=20 \mathrm{~km}$ for all sites and with $\theta_{0}(V L T I, 2.2 \mu \mathrm{m})=13.4 \mathrm{arcsec}$, the highest value of $h_{\max } \theta_{0}$ is obtained for the VLTI: $h_{\max } \theta_{0} \simeq 1.28 \mathrm{~m}$; therefore, for those interferometers, $D>6 h_{\max } \theta_{0}$. Table 3 gives the isopistonic angle values for the KECK, LBT and VLTI interferometers. These values are provided from Eqs. (31) or (32) according to the large or small aperture cases, in $K$ band and for $n=10$ ( $\lambda / 10$ accuracy).

The isopistonic angle values in Table 3 are very low compared to the ones given in Table 4. Indeed, as we will show for Dome $\mathrm{C}$ using a diameter about $8 \mathrm{~m}$, the isopistonic angle values must be higher. This is a severe limitation for the considered interferometers; indeed, when one wants to observe a random faint source, the probability of finding a reference source within the isopistonic angle will be seriously limited.

\subsection{Isopistonic angle at Dome C}

A recent study characterizing the atmosphere at Dome $\mathrm{C}$ (Aristidi 2005) shows that the atmospheric turbulence is principally located in the first $30 \mathrm{~m}$ at this site. The isoplanatic angle is $\theta_{0}(0.5 \mu \mathrm{m}) \simeq 6.8$ arcsec and $\theta_{0}(2.2 \mu \mathrm{m}) \simeq 36.7$ arcsec. This leads to having $h_{\max } \theta_{0} \simeq 0.03 \mathrm{~m}$; so the inequality $D \geq 6 h_{\max } \theta_{0}$ is verified for all aperture diameters $D$ greater than $1 \mathrm{~m}$. Therefore the isopistonic angle at Dome C can be provided by Eqs. (31) or (32).

Table 4 gives the isopistonic angle values at Dome $\mathrm{C}$ for $D=1,2$, and $3 \mathrm{~m}$. This is done in the $K$ band and for $L_{0}=5$, 10 , and $20 \mathrm{~m}$. Calculations were performed using the FRIED parameter value $r_{0}(0.5 \mu \mathrm{m}) \simeq 7.2 \mathrm{~cm}$, or $r_{0}(2.2 \mu \mathrm{m}) \simeq 38.7 \mathrm{~cm}$, which is deduced from the seeing mean value, $\epsilon=1.4$ arcsec, at ground level at Dome C (Agabi et al. 2006), and from the relation: $\epsilon=0.98 \frac{\lambda}{r_{0}}$ (Fried 1966).

One can notice that we reach high values of isopistonic angle at the Dome $\mathrm{C}$ site using a small diameter (1, 2, and $3 \mathrm{~m}$ ), compared to other sites as Paranal. This means that at the Dome $\mathrm{C}$ site an interferometer does not requires a large aperture diameter to reach good sky coverage when observing all directions of the sky. This reinforces the interferometric potential of the Dome C site.

\section{Conclusion}

For the first time, analytical expressions for the anispistonic error and for the isopistonic angle have been found that are valid at the major operational sites. Indeed, using the atmospheric parameters $r_{0}, L_{0}$, and $\theta_{0}$, it is now possible to provide the error due to an off-axis displacement for many control systems, such as a servo-loop fringe tracking system. Our study is also practical for the off-axis guide technique, which is actually the only method of performing long-baseline optical interferometry on faint sources in the near infrared ( $K$ or $H$ band). Furthermore, the off-axis guide is decisive for differential astrometry, which measures angular separation between the target and a reference source, such as PRIMA on the VLTI, for which using a reference star provides the target phase according to the phase-reference imaging technique.

\section{Appendix: Development of the integrals}

Development of the integral $I_{0}(a)$

We recall the expression of the integral $I_{0}(a)$

$I_{0}(a)=a^{-\frac{11}{3}} \int_{0}^{\infty} \frac{J_{1}^{2}(x)}{\left[1+\left(\frac{a}{x}\right)^{-2}\right]^{-11 / 6}} \frac{\mathrm{dx}}{x}$

Using the convolution theorem of MELLIN transform (Sasiela 1994, Eq. (1.27)) and the MELLIN transforms,

$$
\begin{array}{r}
{\left[1+\left(\frac{a}{x}\right)^{-2}\right]^{-11 / 6} \rightarrow \frac{a^{-s}}{2} \frac{\Gamma[-s / 2,11 / 6+s / 2]}{\Gamma(11 / 6)}} \\
-11 / 3<\operatorname{Re}(s)<0
\end{array}
$$

$J_{1}^{2}(x) \rightarrow \frac{1}{2 \sqrt{\pi}} \frac{\Gamma[s / 2+1,1 / 2-s / 2]}{\Gamma[2-s / 2,1-s / 2]}$,

$$
-2<\operatorname{Re}(s)<1,
$$

and replacing $s$ with $4 s$ leads to the new equation

$$
\begin{aligned}
I_{0}(a)= & \frac{a^{-11 / 3}}{\sqrt{\pi} \Gamma(11 / 6)} \frac{1}{2 \mathrm{i} \pi} \int_{C s} \mathrm{~d} s a^{-4 s} \\
& \times \frac{\Gamma[2 s+1,1 / 2-2 s,-2 s, 11 / 6+2 s]}{\Gamma[2-2 s, 1-2 s]},
\end{aligned}
$$

where $s$ is a complex number and $C$ is the integration path in the complex plane.

Sasiela (1994) has shown that this kind of integral can be written as the sum of a series. Each series is the sum of the pole residues of the integrand, so the poles are

(1) $2 s+1=-n$

(2) $2 s+11 / 6=-n$

(3) $1 / 2-2 S=-n$

(4) $-2 s=-n$.

Poles (1) and (2) correspond to the low values of the parameter $a$ : $a<1$. The corresponding series are called TAYLOR series and lead to expression given by Eq. (9). Poles (3) and (4) correspond to high values of $a: a>1$, and the corresponding series are called Asymptotic series and lead to the expression given by Eq. (10). 


\section{Development of the integral $l_{1}(a, b)$}

The integral $I_{1}(a, b)$ is

$I_{1}(a, b)=a^{-\frac{11}{3}} \int_{0}^{\infty} \frac{J_{1}^{2}(x) J_{0}\left(\frac{x}{b}\right)}{\left[1+\left(\frac{a}{x}\right)^{-2}\right]^{\frac{11}{6}}} \frac{\mathrm{dx}}{x}$.

Using the convolution theorem of MELLIN transform (Sasiela 1994, Eq. (1.27)) and the MELLIN transforms, we obtain the correspondence

$J_{0}(x) \rightarrow 2^{-s-1} \frac{\Gamma(-s / 2)}{\Gamma(1+s / 2)}, \quad-3 / 2<\operatorname{Re}(s)<2$.

Using Eqs. (34), (35), and (37), and replacing $s$ with $2 s$ and $t$ with $2 t$ leads to the new expression of the integral $I_{1}(a, b)$ :

$$
\begin{aligned}
I_{1}(a, b)= & \frac{a^{-11 / 3}}{2 \sqrt{\pi} \Gamma(11 / 6)} \frac{1}{(2 \mathrm{i} \pi)^{2}} \int_{C s} \int_{C t} \mathrm{dsdt} \mathrm{a}^{-2 \mathrm{~s}}(2 \mathrm{~b})^{-2 \mathrm{t}} \\
& \times \frac{\Gamma[s+t+1,1 / 2-s-t,-s, 11 / 6+s,-t]}{\Gamma[2-s-t, 1-s-t, 1+t]},
\end{aligned}
$$

Sasiela has shown that integral Eq. (38) can be written as the sum of doubles series (Sasiela 1994), where each series is the sum of an independent 2-pole set of the integrand. There is one series for each combination of the parameters. For integral $I_{1}(a, b)$, the sets of independent 2-poles are
(1) $s+t+1=-n,-s=-m$,
(2) $s+t+1=-, n, 11 / 6+s=-m$,
(3) $s+t+1=-n,-t=-m$,
(4) $1 / 2-s-t=-n,-s=-m$,
(5) $1 / 2-s-t=-n, 11 / 6+s=-m$,
(6) $1 / 2-s-t=-n,-t=-m$,
(7) $-s=-n, \quad-t=-m$,
(8) $11 / 6+s=-n,-t=-m$,

at infinity, the series of the integral converge toward

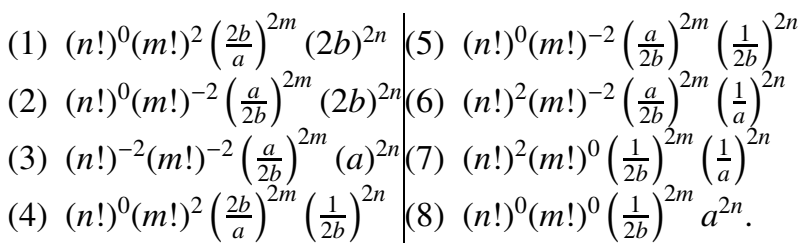

For fast convergence, the no-factorial terms must be less than 1 , and the powers of the factorial must be less than or equal
Table 5. Series ranges for the most rapid convergence.

\begin{tabular}{ccccc}
\hline \hline 2-pole Sets & Solution type & \multicolumn{3}{l}{ Convergence } \\
\hline$(1)$ & Asymptotic & $b<a / 2$ & $\&$ & $b<1 / 2$ \\
$(2)$ & Taylor & $b>a / 2$ & $\&$ & $b<1 / 2$ \\
$(3)$ & Taylor & $a<1$ & $\&$ & $b>a / 2$ \\
$(4)$ & Asymptotic & $b<a / 2$ & $\&$ & $b>1 / 2$ \\
$(5)$ & Taylor & $b>a / 2$ & $\&$ & $b>1 / 2$ \\
$(6)$ & Asymptotic & $a>1$ & $\&$ & $b>a / 2$ \\
$(7)$ & Asymptotic & $a>1$ & $\&$ & $b>1 / 2$ \\
$(8)$ & Taylor & $a<1$ & $\&$ & $b>1 / 2$ \\
\hline
\end{tabular}

to 0 . The powers satisfying this rule lead to convergent series (so-called TAYLOR series), and other series lead to series with asymptotic behavior. In Table 5 we summarize the range of applicability of the series and solution type, which allowed us to obtain Fig. 3.

Acknowledgements. This work benefitted from the efficient help of Mr Marc Sarazin, Rodolphe Heyd, and Abelfettah Habib. This work was also supported by a France-Morocco international collaboration Program, PICS.

\section{References}

Agabi, A., Aristidi, E., Azouit, et al. 2006, PASP, 118, 344

Aristidi, E. 2005, Winter Site Testing at Dome c, Antarctica: first results, in Advanced interferometry, Proc. JENAM

Beckers, J. 1993, ARA\&A, 31, 13

Conan, R., Borgnino, J., Ziad, A., et al. 2000, J. Opt. Soc. Am. A, 17, 1807

Esposito, S., Riccardi, A., \& Femenía, B. 2000, A\&A, 353, L29

Fried, D. L. 1966, J. Opt. Soc. Am., 56, 1372

Goodman, J. W. 2000, Statistical Optics (New york: Wiley Interscience)

Mariotti, J. M. 1994, Adaptive optics for long baseline interferometry, Adaptive Optics for Astronomy, ed. D. M. Alloin, \& J. M. Mariotti (Kluwer Academic Publisher), 309

Parenti, R. R., \& Sasiela, R. J. 1994, J. Opt. Soc. Am. A, 11, 288

Perrin, G. 1997, A\&AS, 121, 553

Quirrenbach, A., Mozourkurkewich, D., Busher, D. F., et al. 1994, A\&A, 286, 1019

Roddier, F. 1981, The Effect of Atmospheric Turbulence in Optical Astronomy, Progress in Optics, XIX, 281

Rodier, F., Gilli, J. M., \& Vernin, J. 1982, J. Opt., Paris, 13, 63

Sarazin, M. 1995, Site Atmopsheric Characterization. in AO'95: Adaptive Optics Topical Meeting, ed. M. Cullum-ESO

Sasiela, R. J. 1994, Electromagnetic Wave Propagation in Turbulence, Evaluation and application of Mellin transforms, in Springer Series on Wave Phenomena, ed. L. M. Brekhovskikh, L. B. Felsen, \& H. A. Haus (SpringerVerlag)

Takato, N., \& Yamaguchi, I. 1995, J. Opt. Soc. Am. Opt. Image Sci. Vision, 12, 958

Tokovinin, A., Vernin, J., Ziad, A., et al. 2005, ASP, 395, 117 